\title{
On the metric dimension of a total graph of non-zero annihilating ideals
}

\author{
Nazi Abachi and Shervin Sahebi
}

\begin{abstract}
Let $R$ be a commutative ring with identity which is not an integral domain. An ideal $I$ of a ring $R$ is called an annihilating ideal if there exists $r \in R-\{0\}$ such that $I r=(0)$. Visweswaran and H. D. Patel associated a graph with the set of all non-zero annihilating ideals of $R$, denoted by $\Omega(R)$ as the graph with the vertex-set $A(R)^{*}$, the set of all non-zero annihilating ideals of $R$ and two distinct vertices $I, J$ are joined if and only if $I+J$ is also an annihilating ideal of $R$. In this paper, we study the metric dimension of $\Omega(R)$ and provide metric dimension formulas for $\Omega(R)$.
\end{abstract}

\section{Introduction}

Assigning a metric dimension to a graph was first introduced by Harary and Melter in [10]. Later, this concept was applied to graphs associated to commutative rings. (see, for example $[7,8,9]$ ). In this paper, we study the metric dimension of a total graph of non-zero annihilating ideals.

Throughout this paper, all rings are assumed to be commutative with identity and they are not fields. The sets of all zero-divisors, nilpotent elements, minimal prime ideals, maximal ideals and Jacobson radical of $R$ are denoted by $Z(R), \operatorname{Nil}(R), \operatorname{Min}(R), \operatorname{Max}(R)$ and $J(R)$, respectively. For a subset $T$ of a ring $R$ we let $T^{*}=T-\{0\}$. An ideal with non-zero annihilator is called an

Key Words: metric dimension, Zero-divisor, Annihilating ideal, Resolving set.

2010 Mathematics Subject Classification: Primary 13A15, 13B99; Secondary 05C99, $05 \mathrm{C} 25$.

Received: 16.09.2019

Accepted: 09.01.2020. 
annihilating ideal. The set of annihilating ideals of $R$ is denoted by $\mathbb{A}(R)$. For every subset $I$ of $R$, we denote the annihilator of $I$ by ann $(I)$. Some more definitions about commutative rings can be find in $[4,5]$.

We use the standard terminology of graphs following [15]. By $G=(V, E)$, we mean a graph, where $V$ and $E$ are the set of vertices and edges, respectively. If we can find at least one path between two any vertices of $G$, then $G$ is called connected. Also, the length of the shortest path between two distinct vertices $x$ and $y$ is denoted by $d(x, y)$ (note that $d(x, y)=\infty$, if there is not any path between $x$ and $y)$ and $\operatorname{diam}(G)=\max \{d(x, y) \mid x, y \in V\}$ is called the diameter of $G$.

The girth of a graph $G$, denoted by $\operatorname{girth}(G)$, is the length of the shortest cycle in $G$. The graph $H=\left(V_{0}, E_{0}\right)$ is a subgraph of $G$ if $V_{0} \subseteq V$ and $E_{0} \subseteq E$. Moreover, $H$ is called an induced subgraph by $V_{0}$, denoted by $G\left[V_{0}\right]$, if $V_{0} \subseteq V$ and $E_{0}=\left\{\{u, v\} \in E \mid u, v \in V_{0}\right\}$. Let $x \in V$, then $N(x)=\{y \in V \mid\{x, y\} \in E\}$ and $N[x]=N(x) \cup\{x\}$.

Let $G=(V, E)$ be a connected graph, $S=\left\{v_{1}, v_{2}, \ldots, v_{k}\right\}$ be an ordered subset of $V$ and $v \in V(G) \backslash S$. The metric representation of $v$ with respect to $S$ is the $k$-vector $D(v \mid S)=\left(d\left(v, v_{1}\right), d\left(v, v_{2}\right), \ldots, d\left(v, v_{k}\right)\right)$. For $S \subseteq V$, if for every $v, u \in V(G)-S, D(u \mid S)=D(v \mid S)$ implies that $u=v$, then $S$ is called the resolving set for $G$. The metric basis for $G$ is a resolving set $S$ of minimum cardinality and the number of elements in $S$ is called the metric dimension of $G\left(\operatorname{dim}_{M}(G)\right)$.

For a graph $G$ with $|V(G)| \geq 2$, if for all $x \in V(G)-\{u, v\}, d(u, x)=d(v, x)$ ( $u, v$ are two distinct vertices), then $u, v$ are distance similar. Clearly, if either $u-v \notin E(G)$ and $N(u)=N(v)$ or $u-v \in E(G)$ and $N[u]=N[v]$, then two distinct vertices $u$ and $v$ are distance similar.

An $k$-partite graph is one whose vertex set can be partitioned into $k$ subsets so that an edge has both ends in no subset. A complete $k$-partite graph is an $k$-partite graph in which each vertex is adjacent to every vertex that is not in the same subset. The complete bipartite (i.e., 2-partite) graph with part sizes $m$ and $n$ is denoted by $K^{m, n}$. If $m=1$, then the bipartite graph is called star graph. A complete graph is a graph such that there exist an edge detween each pair of vertices and is denoted by $K^{n}$.

Let $R$ be a commutative ring with identity which is not an integral domain. An ideal $I$ of a ring $R$ is called an annihilating ideal if there exists $r \in R-\{0\}$ such that $I r=(0)$. Visweswaran and H. D. Patel associated a graph with the set of all non-zero annihilating ideals of $R$, denoted by $\Omega(R)$ as the graph with the vertex-set $A(R)^{*}$, the set of all non-zero annihilating ideals of $R$ and two distinct vertices $I, J$ are joined if and only if $I+J$ is also an annihilating ideal of $R$. In this paper, we study the metric dimension of $\Omega(R)$ and provide metric dimension formulas for $\Omega(R)$. 


\section{Metric dimension of a total graph of a reduced ring}

Let $R$ be a commutative ring. In this section, we provide a metric dimension formula for a total graph of non-zero annihilating ideals when $R$ is reduced.

Lemma 2.1. Suppose that $R$ is a commutative ring with identity and $\Omega(R)$ is connected. If $R$ is not an integral domain, then the following statements are equivalent.

(1) $\operatorname{dim}_{M}(\Omega(R))$ is finite.

(2) $R$ has only finitely many ideals.

Proof. (1) $\Rightarrow(2)$ Assume that $\operatorname{dim}_{M}(\Omega(R))$ is finite and for some non-negative integer $n$, let $W=\left\{I_{1}, I_{2}, \ldots, I_{n}\right\}$ be the metric basis for $\Omega(R)$. Since $\operatorname{diam}(\Omega(R)) \leq 2$ (see [14]), for every $I \in A(R)^{*}-W$, there are only $(2+1)^{n}$ choices for $D(I \mid W)$. So $\left|A(R)^{*}\right| \leq 3^{n}+n$ and hence $R$ has only finitely many ideals.

$$
(2) \Rightarrow(1) \text { is clear. }
$$

If $R$ is a reduced ring with finitely many ideals, then $R$ is Artinian ring and so by [4, Theorem 8.7], $R$ is direct product of finitely many fields. Using this, we prove the following result.

Theorem 2.1. Suppose that $R$ is a reduced ring with identity that is not an integral domain. If $\Omega(R)$ is connected and $\operatorname{dim}_{M}(\Omega(R))$ is finite, then:

(1) If $|\operatorname{Max}(R)|=3$, then $\operatorname{dim}_{M}(\Omega(R))=2$.

(2) If $|\operatorname{Max}(R)|=n \geq 4$, then $\operatorname{dim}_{M}(\Omega(R))=n$.

Proof. (1) If $n=3$, then $R \cong F_{1} \times F_{2} \times F_{3}$, where $F_{i}$ is a field for every $1 \leq i \leq 3$. Now, we put $W=\left\{(0) \times F_{2} \times F_{3}, F_{1} \times(0) \times F_{3}\right\}$ and by Figure 1 , we can easily get

$$
\begin{aligned}
& D\left((0) \times F_{2} \times(0) \mid W\right)=(1,2), \\
& D\left(F_{1} \times(0) \times(0) \mid W\right)=(2,1), \\
& D\left((0) \times(0) \times F_{3} \mid W\right)=(1,1), \\
& D\left(F_{1} \times F_{2} \times(0) \mid W\right)=(2,2) .
\end{aligned}
$$

So for every $x, y \in V(\Omega(R))-W, D(x \mid W) \neq D(y \mid W)$ and hence $\operatorname{dim}_{M}(\Omega(R))=2$. 


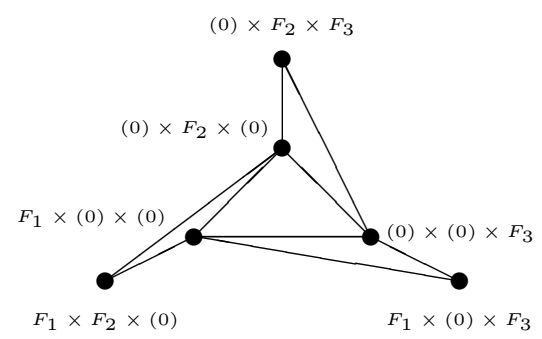

Figure 1: $\Omega\left(F_{1} \times F_{2} \times F_{3}\right)$

(2) Assume that $n \geq 4$. By Lemma $2.1, R \cong F_{1} \times \cdots \times F_{n}$, where $F_{i}$ is a field for every $1 \leq i \leq n$. We show that $\operatorname{dim}_{M}(\Omega(R))=n$. Indeed, we have the following claims:

Claim 1. $\operatorname{dim}_{M}(\Omega(R)) \geq n$.

Since $R$ is direct product of finitely many fields, by Lemma 2.1, $\operatorname{dim}_{M}(\Omega(R))$ is finite. So we can let for some integer $k, W=\left\{I_{1}, I_{2}, \ldots, I_{k}\right\}$ be the metric basis for $\Omega(R)$. On the other hand, since, $\operatorname{diam}(\Omega(R)) \in\{1,2\}$ (see [14]), for every $I \in A(R)^{*}-W$, there are only $2^{k}$ possibilities for $D(I \mid W)$. This implies that $\left|A(R)^{*}\right|-k \leq 2^{k}$. Since $\left|A(R)^{*}\right|=2^{n}-2,2^{n}-2-k \leq 2^{k}$ and hence $2^{n} \leq 2^{k}+2+k$. Since $n \geq 4$, we have $k \geq n$. Therefore, $\operatorname{dim}_{M}(\Omega(R)) \geq n$.

Claim 2. $\operatorname{dim}_{M}(\Omega(R)) \leq n$.

For every $1 \leq i \leq n$, let $\left(I_{1}, I_{2}, \ldots, I_{n}\right)=\mathfrak{m}_{i} \in A(R)^{*}$ such that $I_{i}=0$ and $I_{j}=F_{j}$, for every $1 \leq j \leq n$ with $i \neq j$. We put $W=\left\{\mathfrak{m}_{1}, \mathfrak{m}_{2}, \ldots, \mathfrak{m}_{n}\right\}$ (in fact $W=\operatorname{Max}(R)$ ). We show that $W$ is the resolving set for $\Omega(R)$. For this, let $I, J \in V(\Omega(R))-W$ and $I \neq J$. We need only to show that $D(I \mid W) \neq D(J \mid W)$. Let $I=\left(I_{1}, I_{2}, \ldots, I_{n}\right)$ and $J=\left(J_{1}, J_{2}, \ldots, J_{n}\right)$. Since $I \neq J$, for some $1 \leq i \leq n, I_{i}=0$ and $J_{i}=F_{i}$ or $I_{i}=F_{i}$ and $J_{i}=0$. Without loss of generality, we can assume that $I_{1}=0$ and $J_{1}=F_{1}$. Now, it is easy to see that $d\left(I, \mathfrak{m}_{1}\right)=1$ and $d\left(J, \mathfrak{m}_{1}\right)=2$. This clearly shows that $D(I \mid W) \neq D(J \mid W)$. Therefore $\operatorname{dim}_{M}(\Omega(R)) \leq n$.

Now, by Claim 1, 2 we have $\operatorname{dim}_{M}(\Omega(R))=n$, for $n \geq 4$. 


\section{Metric dimension of a total graph of a non-reduced ring}

In this section, we study the metric dimension of $\Omega(R)$ when $R$ is non-reduced. We begin with the following useful lemma.

Lemma 3.1. Let $R \cong R_{1} \times \cdots \times R_{n}$, where $R_{i}$ is an Artinian local ring for every $1 \leq i \leq n$. and let $I=\left(I_{1}, \ldots, I_{n}\right)$ and $J=\left(J_{1}, \ldots, J_{n}\right)$. Then

(1) $I-J$ is an edge of $\Omega(R)$ if and only if for some $1 \leq i \leq n, I_{i}, J_{i} \subseteq$ $\operatorname{Nil}\left(R_{i}\right)$.

(2) If $0 \neq I \subseteq J(R)$, then $I$ is adjacent to all other vertices in $\Omega(R)$.

Proof. (1) Let $I-J$ be an edge of $\Omega(R)$. If for every $1 \leq i \leq n, I_{i} \not \subseteq \operatorname{Nil}\left(R_{i}\right)$ or $J_{i} \nsubseteq \subseteq \operatorname{Nil}\left(R_{i}\right)$, then for every $1 \leq i \leq n, I_{i}=R_{i}$ or $J_{i}=R_{i}$. This implies that $I+J=R$ and hence $I-J$ is not an edge of $\Omega(R)$, a contradiction. The converse is clear.

(2) By Part (1) is clear.

Remark 3.1. For a connected graph $G$, if $V_{1}, V_{2}, \ldots, V_{k}$ is a partition of $V(G)$ such that for every $1 \leq i \leq k, x, y \in V_{i}$ implies that $N(x)=N(y)$. Then $\operatorname{dim}_{M}(G) \geq|V(G)|-k$.

Theorem 3.1. Suppose that $R \cong R_{1} \times \cdots \times R_{n}$, where $R_{i}$ is an Artinian local ring such that for every $1 \leq i \leq n,\left|A\left(R_{i}\right)^{*}\right| \geq 1$. Then $\operatorname{dim}_{M}(\Omega(R))=$ $\left|A(R)^{*}\right|-2^{n}+1$.

Proof. Suppose that $I=\left(I_{1}, \ldots, I_{n}\right)$ and $J=\left(J_{1}, \ldots, J_{n}\right)$ are vertices of $\Omega(R)$. Define the relation $\sim$ on $V(\Omega(R))$ as follows: $I \sim J$, whenever for each $1 \leq i \leq n, " I_{i} \subseteq \operatorname{Nil}\left(R_{i}\right)$ if and only if $J_{i} \subseteq \operatorname{Nil}\left(R_{i}\right)$ ".

Clearly, $\sim$ is an equivalence relation on $V(\Omega(R))$. The equivalence class of $I$ is denoted by $[I]$. Suppose that $X$ and $Y$ are two elements of the equivalence class of $I$. Since $X \sim Y$, by part (1) of Lemma 3.1, we can easily get $N(X)=$ $N(Y)$. Now, since the number of equivalence classes is $2^{n}-1$, then

$$
\operatorname{dim}_{M}(\Omega(R)) \geq\left|A(R)^{*}\right|-\left(2^{n}-1\right)=\left|A(R)^{*}\right|-2^{n}+1,
$$

by Remark 3.1.

Now, we show that

$$
\operatorname{dim}_{M}(\Omega(R)) \leq\left|Z(R)^{*}\right|-2^{n}+1 .
$$

We put

$$
\begin{aligned}
& A=\left\{\left(I_{1}, \ldots, I_{n}\right) \in V(\Omega(R)) \mid I_{i} \in\left\{0, R_{1}, \ldots, R_{n}\right\} \text { for every } 1 \leq i \leq n\right\} \cup \\
& \left\{\left(\operatorname{Nil}\left(R_{1}\right), \ldots, N i l\left(R_{n}\right)\right)\right\}
\end{aligned}
$$


$W=A(R)^{*}-A$.

We show that $W$ is a resolving set and consequently the metric basis for the graph $\Omega(R)$. For this, let $I, J \in A$ and $I \neq J$. Let $I=\left(I_{1}, \ldots, I_{n}\right)$ and $J=\left(J_{1}, \ldots, J_{n}\right)$. We show that $D(I \mid W) \neq D(J \mid W)$.

We have the following cases:

Case 1. $I=\left(\operatorname{Nil}\left(R_{1}\right), \ldots, N i l\left(R_{n}\right)\right)$ or $J=\left(\operatorname{Nil}\left(R_{1}\right), \ldots, N i l\left(R_{n}\right)\right)$. Without loss of generality, we may assume that $I \neq\left(\operatorname{Nil}\left(R_{1}\right), \ldots, N i l\left(R_{n}\right)\right)$ and $J=\left(N i l\left(R_{1}\right), \ldots, N i l\left(R_{n}\right)\right)$. By Part (2) of Lemma 3.1, we have $D(J \mid W)=$ $(1,1, \ldots, 1)$.To continue the proof let $I^{\prime}=\operatorname{ann}(I)$. Since $I+I^{\prime}=R, I$ is not adjacent to $I^{\prime}$. Since $\left|\left[I^{\prime}\right]\right|>1$ (note that for every $X \in V(\Omega(R)),|[X]|>1$ ), $W \cap\left[I^{\prime}\right] \neq \emptyset$. This implies that for every $K \in W \cap\left[I^{\prime}\right], K$ is not adjacent to $I$ and hence $D(I \mid W) \neq(1,1, \ldots, 1)$. Therefore, $D(I \mid W) \neq D(J \mid W)$.

Case 2. $I \neq\left(\operatorname{Nil}\left(R_{1}\right), \ldots, N i l\left(R_{n}\right)\right)$ and $J \neq\left(\operatorname{Nil}\left(R_{1}\right), \ldots, N i l\left(R_{n}\right)\right)$.

Since $I \nsim J$, for some $1 \leq i \leq n, I_{i}=0$ and $J_{i}=R_{i}$ or $J_{i}=0$ and $I_{i}=R_{i}$. Without loss of generality, we may assume that $I_{1}=0$ and $J_{1}=R_{1}$. So $I=\left(0, I_{2}, \ldots, I_{n}\right)$ and $J=\left(R_{1}, J_{2}, \ldots, J_{n}\right)$. Let $0 \neq K \subseteq N i l\left(R_{1}\right)$ and $X=\left(K, R_{2}, \ldots, R_{n}\right)$. This, clearly follows that, $X \in W, d(I, X)=1$ and $d(J, X)=2$. Therefore, $D(I \mid W) \neq D(J \mid W)$. So

Since $|A|=2^{n}-1$, we have $|W|=\left|A(R)^{*}\right|-\left(2^{n}-1\right)=\left|A(R)^{*}\right|-2^{n}+1$.

$$
\operatorname{dim}_{M}(\Omega(R)) \leq\left|A(R)^{*}\right|-2^{n}+1
$$

Corollary 3.1. Suppose that $R \cong R_{1} \times \cdots \times R_{n}$, where $R_{i}$ is an Artinian local ring such that for every $1 \leq i \leq n,\left|A\left(R_{i}\right)^{*}\right|=1$. Then $\operatorname{dim}_{M}(\Omega(R))=$ $3^{n}-2^{n}-1$.

Lemma 3.2. Let $R \cong R_{1} \times \cdots \times R_{n} \times F_{1} \times \cdots \times F_{m}, n \geq 1, m \geq 1$ where each $\left(R_{i}, \mathfrak{m}_{i}\right)$ is an Artinian local ring with $\mathfrak{m}_{i} \neq(0)$ and each $F_{i}$ is a field and let $S \cong F_{1}^{\prime} \times \cdots \times F_{n+m}^{\prime}$ where each $F_{i}^{\prime}$ is a field. Then $\operatorname{dim}_{M}(\Omega(R)[A])=\operatorname{dim}_{M}(\Omega(S))=m+n$, where $A=\left\{\left(I_{1}, \ldots, I_{n+m}\right) \in\right.$ $\left.V(\Omega(R)) \mid I_{i} \in\left\{0, R_{1}, \ldots, R_{n}, F_{1}, \ldots, F_{m}\right\}\right\} \cup\left\{\left(\operatorname{Nil}\left(R_{1}\right), \ldots, N i l\left(R_{n}\right), 0, \ldots, 0\right)\right\}$.

Proof. It is not hard to see that $\Omega(R)[A] \cong \Omega(S)$ and hence by Theorem 2.1, $\operatorname{dim}_{M}(\Omega(R)[A])=\operatorname{dim}_{M}(\Omega(S))=m+n$.

Theorem 3.2. Let $R \cong R_{1} \times \cdots \times R_{n} \times F_{1} \times \cdots \times F_{m}$, be a finite ring, $n \geq 1$, $m \geq 1$ where each $\left(R_{i}, \mathfrak{m}_{i}\right)$ is an Artinian local ring with $\mathfrak{m}_{i} \neq(0)$ and each $F_{i}$ is a field. Then $\operatorname{dim}_{M}(\Omega(R))=\left|A(R)^{*}\right|-2^{n+m}+m+1$.

Proof. Assume that $I=\left(I_{1}, \ldots, I_{n}\right)$ and $J=\left(J_{1}, \ldots, J_{n}\right)$ are vertices of $\Omega(R)$. Define the relation $\sim$ on $V(\Omega(R)$ ) as follows: $I \sim J$, whenever for every $1 \leq i \leq n, " I_{i} \subseteq \operatorname{Nil}\left(R_{i}\right)$ if and only if $J_{i} \subseteq \operatorname{Nil}\left(R_{i}\right)$ ". 
Clearly, $\sim$ is an equivalence relation on $V(\Omega(R))$. The equivalence class of $I$ is denoted by $[I]$. Suppose that $X$ and $Y$ are two elements of the equivalence class of $I$. Since $X \sim Y$, by part (2) of Lemma 3.1, we can easily get $N(X)=$ $N(Y)$. Now, since the number of equivalence classes is $2^{n+m}-1$, then

$$
\operatorname{dim}_{M}(\Omega(R)) \geq\left|A(R)^{*}\right|-\left(2^{n+m}-1\right)=\left|A(R)^{*}\right|-2^{n+m}+1
$$

by Remark 3.1. Now, we put

$A=\left\{\left(I_{1}, \ldots, I_{n+m}\right) \in V(\Omega(R)) \mid I_{i} \in\left\{0, R_{1}, \ldots, R_{n}, F_{1}, \ldots, F_{m}\right\}\right\} \cup$

$\left\{\left(\operatorname{Nil}\left(R_{1}\right), \ldots, \operatorname{Nil}\left(R_{n}\right), 0, \ldots, 0\right)\right\}$.

In fact, $|A|=2^{n+m}-1$ and for every equivalence class say $[I],|[I] \cap A|=1$.

This means that if $W$ is a resolving set for $\Omega(R)$, then $A(R)^{*}-A \subseteq W$.

Also, for every $n+1 \leq i \leq n+m$, let

$$
K_{i}=\left(R_{1}, R_{2}, \ldots, R_{n}, J_{n+1}, J_{n+2}, \ldots, J_{n+m}\right)
$$

such that $J_{j}=0$ if $i=j$, and if $i \neq j, J_{j}=F_{j}$ and let

$B=\left\{K_{n+1}, K_{n+2}, \ldots, K_{n+m}\right\}$

$C=\left\{\left(0,0, \ldots, 0, I_{n+1}, \ldots, I_{n+m}\right) \in V(\Omega(R)) \mid I_{n+j} \in\right.$

$\left\{0, F_{1}, \ldots, F_{m}\right\}$ for every $\left.1 \leq j \leq m\right\} \cup\left\{\left(\operatorname{Nil}\left(R_{1}\right), \ldots, N i l\left(R_{n}\right), 0, \ldots, 0\right)\right\}$.

Now, let $I=\left(I_{1}, \ldots, I_{n+m}\right) \in A(R)^{*}-A \subseteq W$. Then since $|[I]|>1$, for some $1 \leq i \leq n, I_{i} \subseteq \operatorname{Nil}\left(R_{i}\right)$. So by Lemma 3.1, every element of $A(R)^{*}-A$ is adjacent to all elements of $C \subseteq A$. Thus we need to add some elements of $A$ to $A(R)^{*}-A$ to get $W$. For this by proof of Theorem 2.1 for reduced rings and Lemma 3.2, the best candidate is the set of elements of $B$. So $B \subseteq W$. This implies that

$$
\operatorname{dim}_{M}(\Omega(R)) \geq\left|A(R)^{*}\right|-2^{n+m}+m+1 .
$$

We prove that $W=\left\{A(R)^{*}-A\right\} \cup B$ is a resolving set and consequently the metric basis for the graph $\Omega(R)$. But this is clear by Theorem 2.1 and Lemma 3.2 .

We close this section with the following example which is related to Theorem 3.2 .

\section{Example 3.1.}

(1) Let $R=\mathbb{Z}_{4} \times \mathbb{Z}_{2}$. Then $n=m=1$ (in Theorem 3.2). We have $\left|A(R)^{*}\right|=4$. By Theorem 3.2, $\operatorname{dim}_{M}(\Omega(R))=\left|A(R)^{*}\right|-2^{n+m}+m+1=4-$ $2^{2}+1+1=2$. Also, by the following figure, we regain that $\operatorname{dim}_{M}(\Omega(R))=2$. 


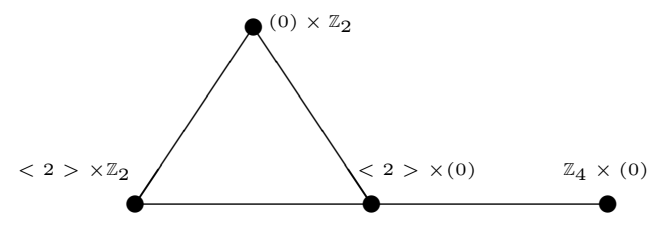

$\Omega\left(\mathbb{Z}_{4} \times \mathbb{Z}_{2}\right)$

(2) Let $R=\mathbb{Z}_{4} \times \mathbb{Z}_{4} \times \mathbb{Z}_{2} \times \mathbb{Z}_{2}$. Then $n=m=2$ (in Theorem 3.2). We have $\left|A(R)^{*}\right|=34$.

$A=\left\{\left(0, \mathbb{Z}_{4}, \mathbb{Z}_{2}, \mathbb{Z}_{2}\right),\left(\mathbb{Z}_{4}, 0, \mathbb{Z}_{2}, \mathbb{Z}_{2}\right),\left(\mathbb{Z}_{4}, \mathbb{Z}_{4}, 0, \mathbb{Z}_{2}\right),\left(\mathbb{Z}_{4}, \mathbb{Z}_{4}, \mathbb{Z}_{2}, 0\right),\left(0,0, \mathbb{Z}_{2}, \mathbb{Z}_{2}\right)\right.$,

$\left(0, \mathbb{Z}_{4}, 0, \mathbb{Z}_{2}\right),\left(0, \mathbb{Z}_{4}, \mathbb{Z}_{2}, 0\right),\left(\mathbb{Z}_{4}, 0, \mathbb{Z}_{2}, 0\right),\left(\mathbb{Z}_{4}, 0,0, \mathbb{Z}_{2}\right),\left(\mathbb{Z}_{4}, \mathbb{Z}_{4}, 0,0\right),\left(\mathbb{Z}_{4}, 0,0,0\right)$,

$\left.\left(0, \mathbb{Z}_{4}, 0,0\right),\left(0,0, \mathbb{Z}_{2}, 0\right),\left(0,0,0, \mathbb{Z}_{2}\right),((2),(2), 0,0)\right\}$.

Since $A(R)^{*}=\cup_{I \in A}[I]$ and for every $X, Y \in[I], N(X)=N(Y)$, we must have $A(R)^{*}-A \subseteq W$ (in fact $X \in W$ or $Y \in W$ ). Now, let

$B=\left\{\left(\mathbb{Z}_{4}, \mathbb{Z}_{4}, \mathbb{Z}_{2}, 0\right),\left(\mathbb{Z}_{4}, \mathbb{Z}_{4}, 0, \mathbb{Z}_{2}\right)\right\}$

$C=\left\{\left(0,0,0, \mathbb{Z}_{2}\right),\left(0,0, \mathbb{Z}_{2}, 0\right),\left(0,0, \mathbb{Z}_{2}, \mathbb{Z}_{2}\right),((2),(2), 0,0)\right\}$.

So by Lemma 3.1, every element of $A(R)^{*}-A$ is adjacent to all elements of $C$ and since $|C|=4$, we need to add at least two elements of $A$ to $A(R)^{*}-A$ to get $W$. So we can let $B \subseteq W$. Therefore,

$W=\left\{\left((2), \mathbb{Z}_{4}, \mathbb{Z}_{2}, \mathbb{Z}_{2}\right),\left(\mathbb{Z}_{4},(2), \mathbb{Z}_{2}, \mathbb{Z}_{2}\right),\left((2),(2), \mathbb{Z}_{2}, \mathbb{Z}_{2}\right),\left((2),(2), 0, \mathbb{Z}_{2}\right)\right.$,

$\left((2),(2), \mathbb{Z}_{2}, 0\right),\left((2), \mathbb{Z}_{4}, 0, \mathbb{Z}_{2}\right),\left((2), \mathbb{Z}_{4}, \mathbb{Z}_{2}, 0\right),\left(\mathbb{Z}_{4},(2), \mathbb{Z}_{2}, 0\right),\left(\mathbb{Z}_{4},(2), 0, \mathbb{Z}_{2}\right)$,

$\left(\mathbb{Z}_{4}, \mathbb{Z}_{4}, \mathbb{Z}_{2}, 0\right),\left(\mathbb{Z}_{4}, \mathbb{Z}_{4}, 0, \mathbb{Z}_{2}\right),\left(\mathbb{Z}_{4},(2), 0,0\right),\left((2), \mathbb{Z}_{4}, 0,0\right),\left((2),(0), \mathbb{Z}_{2}, \mathbb{Z}_{2}\right)$,

$\left((2), 0,0, \mathbb{Z}_{2}\right),\left((2), 0, \mathbb{Z}_{2}, 0\right),\left(0,(2), \mathbb{Z}_{2}, \mathbb{Z}_{2}\right),\left(0,(2), 0, \mathbb{Z}_{2}\right),\left(0,(2), \mathbb{Z}_{2}, 0\right)$,

$((2), 0,0,0),(0,(2), 0,0)\}$.

We have:

$D\left(\left(0, \mathbb{Z}_{4}, \mathbb{Z}_{2}, \mathbb{Z}_{2}\right) \mid W\right)=(1,2,1,1,1,1,1,2,2,2,2,2,1,1,1,1,1,1,1,1,1)$,

$D\left(\left(\mathbb{Z}_{4}, 0, \mathbb{Z}_{2}, \mathbb{Z}_{2}\right) \mid W\right)=(2,1,1,1,1,2,2,1,1,2,2,1,2,1,1,1,1,1,1,1,1)$,

$D\left(\left(0,0, \mathbb{Z}_{2}, \mathbb{Z}_{2}\right) \mid W\right)=(1,1,1,1,1,1,1,1,1,2,2,1,1,1,1,1,1,1,1,1,1)$,

$D\left(\left(0, \mathbb{Z}_{4}, 0, \mathbb{Z}_{2}\right) \mid W\right)=(1,2,1,1,1,1,1,2,1,2,1,1,1,1,1,1,1,1,1,1,1)$,

$D\left(\left(0, \mathbb{Z}_{4}, \mathbb{Z}_{2}, 0\right) \mid W\right)=(1,2,1,1,1,1,1,1,2,1,2,1,1,1,1,1,1,1,1,1,1)$,

$D\left(\left(\mathbb{Z}_{4}, 0, \mathbb{Z}_{2}, 0\right) \mid W\right)=(2,1,1,1,1,2,1,1,1,1,2,1,1,1,1,1,1,1,1,1,1)$,

$D\left(\left(\mathbb{Z}_{4}, 0,0, \mathbb{Z}_{2}\right) \mid W\right)=(2,1,1,1,1,1,2,1,1,2,1,1,1,1,1,1,1,1,1,1,1)$,

$D\left(\left(\mathbb{Z}_{4}, \mathbb{Z}_{4}, 0,0\right) \mid W\right)=(2,2,2,1,1,1,1,1,1,1,1,1,1,2,1,1,2,1,1,1,1)$,

$D\left(\left(\mathbb{Z}_{4}, 0,0,0\right) \mid W\right)=(2,1,1,1,1,1,1,1,1,1,1,1,1,1,1,1,1,1,1,1,1)$,

$D\left(\left(0, \mathbb{Z}_{4}, 0,0\right) \mid W\right)=(1,2,1,1,1,1,1,1,1,1,1,1,1,1,1,1,1,1,1,1,1)$,

$D\left(\left(0,0, \mathbb{Z}_{2}, 0\right) \mid W\right)=(1,1,1,1,1,1,1,1,1,1,2,1,1,1,1,1,1,1,1,1,1)$,

$D\left(\left(0,0,0, \mathbb{Z}_{2}\right) \mid W\right)=(1,1,1,1,1,1,1,1,1,2,1,1,1,1,1,1,1,1,1,1,1)$, 
$D(((2),(2), 0,0) \mid W)=(1,1,1,1,1,1,1,1,1,1,1,1,1,1,1,1,1,1,1,1,1)$.

It is seen that for every $I, J \in A(R)^{*}-W$ with $I \neq J, D(I \mid W) \neq D(J \mid W)$

and so $W$ is a resolving set and consequently the metric basis for the graph $\Omega\left(\mathbb{Z}_{4} \times \mathbb{Z}_{4} \times \mathbb{Z}_{2} \times \mathbb{Z}_{2}\right)$.

\section{References}

[1] D.F. Anderson, P.S. Livingston, The zero-divisor graph of a commutative ring, J. Algebra, 217 (1999), 434-447.

[2] A. Badawi, On the annihilator graph of a commutative ring, Comm. Algebra, 42 (2014), 108-121.

[3] A. Badawi, On the dot product graph of a commutative ring, Comm. Algebra, 43 (2015), 43-50.

[4] M.F. Atiyah, I.G. Macdonald, Introduction to Commutative Algebra, Addison-Wesley Publishing Company, (1969).

[5] W. Bruns, J. Herzog, Cohen-Macaulay Rings, Cambridge University Press, (1997).

[6] N. Ganesan, Properties of rings with a finite number of zero-divisors, Math. Ann., 157 (1964), 215-218.

[7] S. Pirzada, R. Raja, S. P. Redmond, Locating sets and numbers of graphs associated to commutative rings, J. Algebra Appl., 13:7 (2014), 1450047 (18pp).

[8] S. Pirzada, R. Raja, On the metric dimension of a zero-divisor graph, Comm. Algebra, 45:4 (2017), 1399-1408.

[9] R. Raja, S. Pirzada, S. P. Redmond, On Locating numbers and codes of zero-divisor graphs associated with commutative rings, J. Algebra Appl., 15:1 (2016), 1650014 (22pp).

[10] F. Harary, R. A. Melter, On the metric dimension of a graph, Ara Combin., 2 (1976), 191-195.

[11] R. Nikandish, M.J. Nikmehr, M. Bakhtyiari, Coloring of the annihilator graph of a commutative ring, J. Algebra Appl., 15:6 (2016), 1650124 (13 $\mathrm{pp})$.

[12] J. A. Huckaba, Commutative Rings with Zero Divisors, 2nd ed., Prentice Hall, Upper Saddle River, (1988). 
[13] T. Y. Lam, A First Course in Non-Commutative Rings Springer-Verlag, New York, Inc, (1991).

[14] S. Visweswaram, H. D. Patel, A graph associated with the set of all nonzero annihilating ideals of a commutative Ring, Discrete Mathematics, Algorithms and Applications, 6:4 (2014), 1450047 (22 pp).

[15] D.B. West, Introduction to Graph Theory, 2nd ed, Prentice Hall, Upper Saddle River, (2001).

Nazi Abachi,

Department of Mathematics,

Central Tehran Branch, Islamic Azad University

Tehran, Iran, P. O. Box 14168-94351.

Email: n_abachi@yahoo.com

Shervin Sahebi,

Department of Mathematics,

Central Tehran Branch, Islamic Azad University

Tehran, Iran, P. O. Box 14168-94351.

Email: sahebi@iauctb.ac.ir 